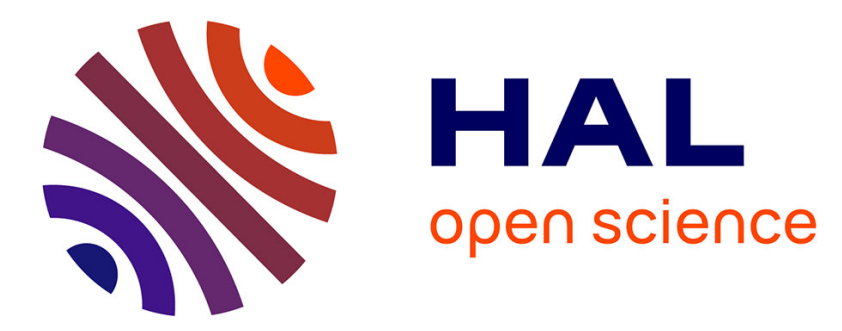

\title{
Tactile Sensors Based Steering as a Substitute of the Attendant Joystick in Powered Wheelchairs
}

\author{
Andres Trujillo-Leon, Wael Bachta, Fernando Vidal-Verdú
}

\section{To cite this version:}

Andres Trujillo-Leon, Wael Bachta, Fernando Vidal-Verdú. Tactile Sensors Based Steering as a Substitute of the Attendant Joystick in Powered Wheelchairs. IEEE Transactions on Neural Systems and Rehabilitation Engineering, 2018, 26 (7), pp.1381-1390. 10.1109/TNSRE.2018.2838326 . hal02492148

\section{HAL Id: hal-02492148 \\ https://hal.sorbonne-universite.fr/hal-02492148}

Submitted on 26 Feb 2020

HAL is a multi-disciplinary open access archive for the deposit and dissemination of scientific research documents, whether they are published or not. The documents may come from teaching and research institutions in France or abroad, or from public or private research centers.
L'archive ouverte pluridisciplinaire HAL, est destinée au dépôt et à la diffusion de documents scientifiques de niveau recherche, publiés ou non, émanant des établissements d'enseignement et de recherche français ou étrangers, des laboratoires publics ou privés. 


\title{
Tactile Sensors Based Steering as a Substitute of the Attendant Joystick in Powered Wheelchairs
}

\author{
Andrés Trujillo-León, Wael Bachta and Fernando Vidal-Verdú
}

\begin{abstract}
Attendant joysticks of powered wheelchairs are devices oriented to help caregivers. Diseases and disabilities such as dementia, spinal cord injuries or blindness make the user unable to drive the chair by his or her own. However, this device is not intuitive to use, especially for old people. Proper processing of the information provided by two tactile sensors in the handlebars achieves control signals that allow an easy and intuitive driving. This is done in this paper, where the performance of this approach is evaluated in comparison with that of the joystick by means of objective measurements as well as questionnaires to obtain the subjective perception of the participants in the experiments. The results show a better performance of the handlebar in terms of error in following a trajectory, collisions with the surrounding furniture, and user feeling related to ease of use, comfort, required training, usefulness, safety and fatigue.
\end{abstract}

Index Terms-Assistive Technology, Tactile Sensors, Wheelchairs, Joystick.

\section{INTRODUCTION}

D EVELOPED societies are getting old, so that facing the increasing aging of their inhabitants must be a crucial goal. In the coming decades, the predicted population pyramid for these countries will lose their triangular shape [1]. From 2000 until 2050, the world's population aged 60 and over will more than triple from 600 million to 2 billion [2]. Aging well requires health and functional capacity in the daily life activity [3]. Inside EU-27, the $52.9 \%$ of disabled people reported that their incapacity causes them mobility difficulties [4]. This is one of the main barriers to participation in daily life activities. In this context, assistive technology becomes a key piece in terms of quality life improvement. When users can walk, the more commonly used assistive tool to increase gait stability are canes and walkers [5], [6]. However, there is a numerous group of people with limited walking ability that may require other kind of mobility aid. For example, certain post-stroke patients [7], some persons affected by cerebral palsy [8] or the elderly [9], especially the oldest old, that is a group with high rates of physical and cognitive impairment [10]. In this sense, powered wheelchairs

This work was partially funded by the Spanish Government under contract TEC2015-67642-R, FPU Program and European ERDF funds. It has also been partly accomplished within the laboratory of excellence SMART supported by French state funds managed by the ANR within the Investissements d'Avenir programme under reference ANR-11-IDEX-0004-02.

A. Trujillo-León and F. Vidal-Verdú are with the Departament of Electronics at the University of Málaga and the Institute of Biomedical Research of Málaga (IBIMA), Málaga, Spain (atrujilloleon@uma.es, fvidaleuma.es).

W. Bachta is with CNRS, UMR 7222, Institut des Systèmes Intelligents et de Robotique, UPMC Univ Paris 06, Paris, France (wael.bachta@upmc.fr).
(PW) are reported as a means of extending the activity and participation of their users [11]. They are usually driven with a hand-operated joystick located at the end of the armrest. Although PWs provide a valuable support, there are people who may not be able to operate theses devices by themselves. Clinicians reported that $9-10 \%$ of patients find them extremely difficult or impossible to use in their daily life. Regarding maneuvering tasks, a $40 \%$ find it impracticable. $85 \%$ of clinicians see every year patients not able to use a PW since they lack the necessary motor skills, strength o visual sharpness. $\mathrm{Up}$ to $9 \%$ of individuals require the assistance of another person [12]. Experienced users also report having difficulties performing basic tasks that involve controlling the joystick and maneuvering [13]. Researchers have contributed with many instruments aimed at helping people to drive wheelchairs [14][24]. They are mainly either joystick variations or alternative driving systems. However, the assistance of a caregiver to propel the chair is required in cases of severe limitations: dementia, acute cerebral palsy, Alzheimer, visual impairment, etc. Since propelling a wheelchair in day to day life is a hard task that may cause health problems [25], it is useful that assistants can benefit from PWs advantages. The common commercial driving solution aimed at assistants consists in another joystick placed at the rear part of the chair [26]. However, as has been reported, this device is not very comfortable and intuitive. Regarding research centered on the caregiver, authors of [27] present an assisting controller based on the force-velocity relationship. It generates an assisting force when the attendant's propelling force exceeds a threshold. However, it does not contemplate steering and only simulations results are presented. Kakimoto et al. propose a prototype of a power-assisted attendant propelled wheelchair that detects the caregiver propulsion force through a force sensor placed on the shaft of the handling bar [28]. Although the impulsion force is reduced by 50 to $60 \%$, the behavior is unstable under certain conditions. It was not intended to help in steering but only in climbing ramps. A power assist system for omnidirectional wheelchairs is exposed in [29], where the input force of the attendant is measured by six-axis force sensor attached to the handle of the chair. The work presented in [30] is a combination of wheelchair and walker, where an admittance based controller has proven to have a good performance controlling the pushing force, acquired through a force sensor placed between the handle and the robot structure. In the work presented in [31], the authors combine the user input, acquired by two force sensors, with an estimation of the environment disturbance to implement an adaptive force control. Most proposals infer the carer driving intention trough 
a haptic interface based on costly force sensors, that are a barrier to market entry [32].

Tactile sensors could be an alternative to the latter in haptic devices. Simple tactile sensors can be built at a low cost [33]. Besides, these sensors have other possible advantages. For instance, the same sensor can implement a panel touch as user interface. They also can gather information about the grip for rehabilitation or ergonomic purposes [34]. Moreover, as a kind of artificial skin, they can be integrated in a system without significant modifications that are likely unavoidable in the case of force sensors. In summary, it is worth exploring this technology as an alternative to joysticks and force sensors. In [35] the authors presented a tactile handlebar aimed at assistants with the purpose of providing them with an easy and effective solution to drive PWs. The main idea was to activate the PW engines according to the carer intention. A basic control algorithm was used to test the viability of the system. Although the preliminary results were not bad, some flaws were detected. The most relevant was that the assistant needed to be instructed to use the system, which decreased the intuitiveness. A different strategy is followed in this paper, that is based on the observed correlation between variables obtained from the tactile sensor based haptic handlebar and forces exerted on it. Force sensors to measure force and torque can then be replaced by tactile sensors and a simple control procedure can be implemented that results in a much more intuitive driving. A thorough evaluation of this approach is also presented. It consisted of two experiments. In the first one, a custom optical based tracking system was developed to measure the error of the user in following a defined trajectory. This provides objective data of the system performance.

Besides, a second test was carried out that consisted in driving in an enclosed indoor space trying not to collide with the environment. Both tests were performed also using a commercial joystick, which is the most common alternative. Moreover, the assessing is also focused on gathering the subjective perception and the opinion of the participants. This way, they were asked to fill a questionnaire answering several questions about their experience using both driving systems. The results showed an overall good performance of the haptic handlebar, that is better than that obtained for the attendant joystick.

This paper is structured as follows. BLABLABLA (estructura del paper, a escribir cuando esté versión más definitiva)

\section{HAPTIC HANDLEBAR TO ASSIST WHEELCHAIRS PROPELLING}

Firstly, the haptic handlebar implementation is explained. Then, the experimental setup designed to its study is detailed.

\section{A. Device}

Since it intends to be intuitive, the tactile handlebar should be used in the same way as a regular one. It is comprised of two handles, one for each hand, covered by rectangular commercial force sensing resistor sensors (FSR) [36]. Figure 1 shows the device schematic and Figure 2 its implementation. The pressure sensors are organized in the form of a matrix of $8 \times 2$ tactels. This configuration minimizes the addressing resources needed to scan the arrays of the left and the right handle. The rows and columns are connected to a custom conditioning electronics board based on a PIC18F4680 microcontroller. This way, the reading process is as follows: a set of analog switches activates the row to be read. Then, the resistance variation in each tactel of the selected row is turned into a voltage variation by transimpedance amplifiers.

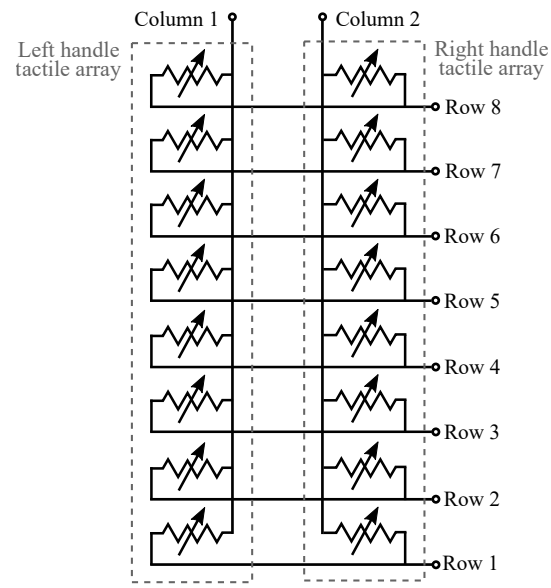

Fig. 1. Tactile sensors schematic. Column 1 corresponds to the tactile sensor that covers the left handle and column 2 to that that covers the right handle.

Finally, the amplifiers output is digitized by the analog-todigital converter of the PIC18F4680. The microcontroller can send the pressure data to a computer via USB. More details can be found in [35].

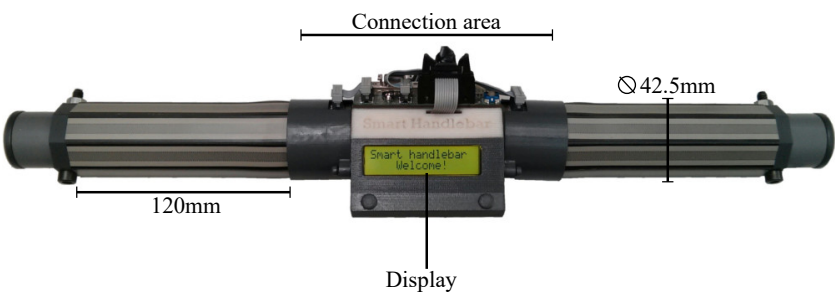

Fig. 2. Tactile handlebar implementation.

\section{B. Control variables}

The pair of control variables used to compute the hapticbased PW movement are based on the centers of mass $(C o M)$ of the pressure maps captured by the left and the right handle tactile sensors. The center of mass (also known as center of pressure) is often used in the processing of tactile images in robotic manipulation tasks. It summarizes the information about how the pressure distribution is in a single spatial coordinate. In the case of this device, it is calculated for the left and right handle sensors as indicated in Equation 1.

$$
C o M_{L / R}=\frac{\sum_{i=1}^{8} i \cdot p(i)}{\sum_{i=1}^{8} p(i)}
$$

where $i$ and $p(i)$ are the position (row) and the pressure value of the $i^{t h}$ tactel in the handle for which $C o M$ is being calculated. $C o M_{L}$ and $C o M_{R}$ are calculated using 
the column 1 and 2, respectively, of the schematic shown in Figure 1.

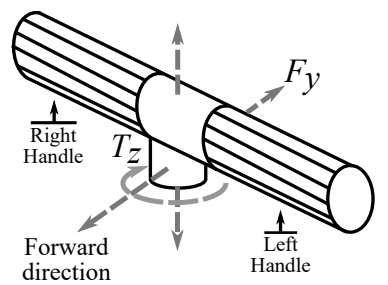

Fig. 3. Location of $F_{y}$ and $T_{z}$, force and torque that model the user interaction when driving the $\mathrm{PW}$ grasping the handlebar.

During driving, pushing/pulling maneuvers can be modeled as a force vector in forward direction $\left(F_{y}\right)$. On the other hand, turns can be defined as the generation of a torque $\left(T_{z}\right)$ in the vertical axis through the middle of the handlebar and perpendicular to the floor. Both of them are illustrated in Figure 3. The sum and subtraction of the centers of mass of the left $\left(C o M_{L}\right)$ and right $\left(C o M_{R}\right)$ handles computed as shown in Equation 2 are highly correlated with $F_{y}$ and $T_{z}$, respectively [37].

$$
\begin{aligned}
S U M_{C o M} & =C_{o} M_{L}+C_{o} M_{R} \\
S U B_{C o M} & =C o M_{L}-C_{o} M_{R}
\end{aligned}
$$

Figure 4 illustrates this correlation by showing $S U M_{C o M}$, $S U B_{C o M}, F_{y}$ and $T_{z}$ registered in a test where the handlebar is manipulated.
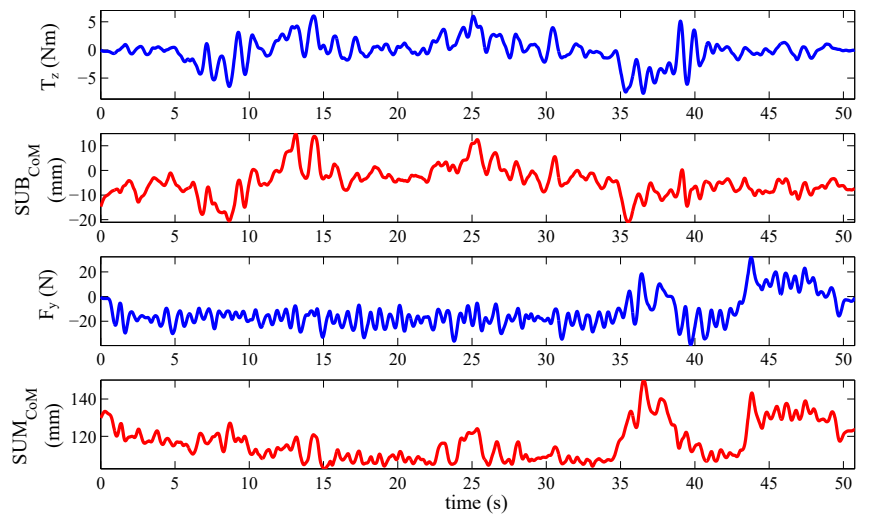

Fig. 4. Parameters captured while using the handlebar. From top to bottom: $T_{z}, S U B_{C o M}, F_{y}$ and $S U M_{C o M}$.

This way, pushing, pulling and turning maneuvers may be identified and quantified using exclusively these two parameters extracted from the tactile sensors.

Specifically, the target linear and angular velocities of the PW $(v, \omega)$ are defined as:

$$
\begin{gathered}
v=G_{l i n} S U M_{C o M} \\
\omega=G_{\text {ang }} S U B_{C o M}
\end{gathered}
$$

where $G_{l i n}$ and $G_{a n g}$ may be used to tune the sensibility according to the assistant preferences.

The variables in Equation 2 have to be referred to their values at the starting point when the user grasps the handles.
Otherwise, $S U M_{C o M}$ would not be zero and the chair would move ( $v \neq 0$ in Equation 3 ) by just holding the handles. In addition, since there is not a perfect match of both the left and right handles and the grip with both hands can also be different, $S U B_{C o M}$ could be a non zero value and $\omega \neq 0$ in Equation 3 despite the user has no intention to turn.

In this way, a dynamic calibration has to be carried out every time the handlebar is grasped. The procedure is as follows: when the grip is detected, the centers of mass in rest condition $\left(\mathrm{CoM}_{L_{r}}\right.$ and $\left.\mathrm{CoM}_{R_{r}}\right)$ are computed. This situation corresponds to the previously described scenario, in which the caregiver just grasps the handles without exerting additional forces aimed at driving the PW. Once both parameters are known and stored, the user receives an audible signal that indicates that he or she can start driving. The procedure takes around one second. Afterwards, during the movement processing, the CoM is computed as shown in Equation 4.

$$
C o M=\operatorname{CoM}_{m}-\operatorname{CoM}_{r}
$$

where $C o M_{m}$ is the center of mass computed for the measured tactile image and $\mathrm{CoM}_{r}$ is that center of mass computed in rest condition in the calibration process. Note that $\operatorname{CoM}_{r}$ becomes a zero reference for the value of the computed $C o M$, so that it will be positive or negative depending on whether it is above or below $\mathrm{CoM}_{r}$.

Considering the above, the control variables could be expressed as:

$$
\begin{gathered}
S U M_{C o M}=S U M_{C o M_{m}}-S U M_{C o M_{r}} \\
S U B_{C o M}=S U B_{C o M_{m}}-S U B_{C o M_{r}}
\end{gathered}
$$

where $S U M_{C o M_{m}}$ and $S U B_{C o M_{m}}$ are the addition and subtraction of $C o M_{L_{m}}$ and $C o M_{R_{m}}$, from Equation 4.

With the latter modifications, $v$ and $\omega$ are zero and the PW remains stopped while the handlebar is grasped unless forces are intentionally exerted.

\section{EXPERIMENTAL SETUP}

The scheme of the experimental setup is shown in Figure 5. The haptic handlebar is attached to the back of the chair frame so that it can be grasped by the assistant to propel the PW. The conditioning electronics previously described scans the tactile sensors and sends the maps of pressure to a computer via USB. The latter calculates $C o M_{L}$ and $C o M_{R}$ using the pressure information of each handle and, from these parameters, the control variables $S U M_{C o M}$ and $S U B_{C o M}$ are computed. Once the target linear and angular velocities are estimated (see Equation 3), the correspondent signals are converted into their analog equivalent through a NI USB6009 multifunction card connected to the computer. The analog outputs of this card are wired into the joystick socket of the master control module REM24SD that the PW incorporates at the end of the right armrest. This is because the activation of the engines is achieved by emulating the output signals of the joystick included in the module REM24SD. The REM24SD, in turn, communicates with the PW power module that supplies the engines. The chair velocities are finally updated at a rate of $60 \mathrm{~Hz}$. 


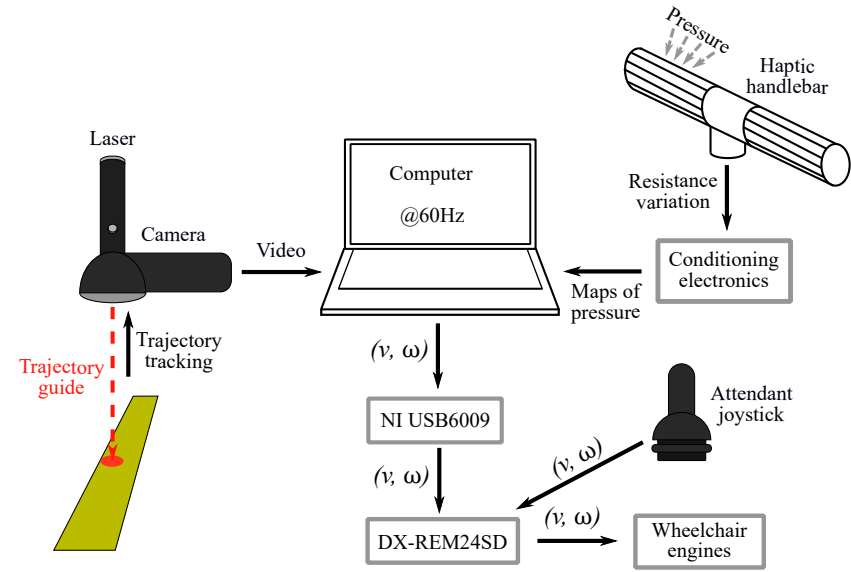

Fig. 5. Experimental setup scheme.

In addition, an attendant joystick is placed in the chair frame near the handlebar and it is also connected to the joystick socket of the master control module. Thus, the PW can be driven by the caregiver using both devices. To register the error in following a certain trajectory, a laser pointer plus a Logitech Pro 9000 camera are mounted on the front of the chair. The implementation of the experimental setup is shown in Figure 6. The whole setup weighs approximately the same as a manual wheelchair with a $80 \mathrm{~kg}$ person on it.

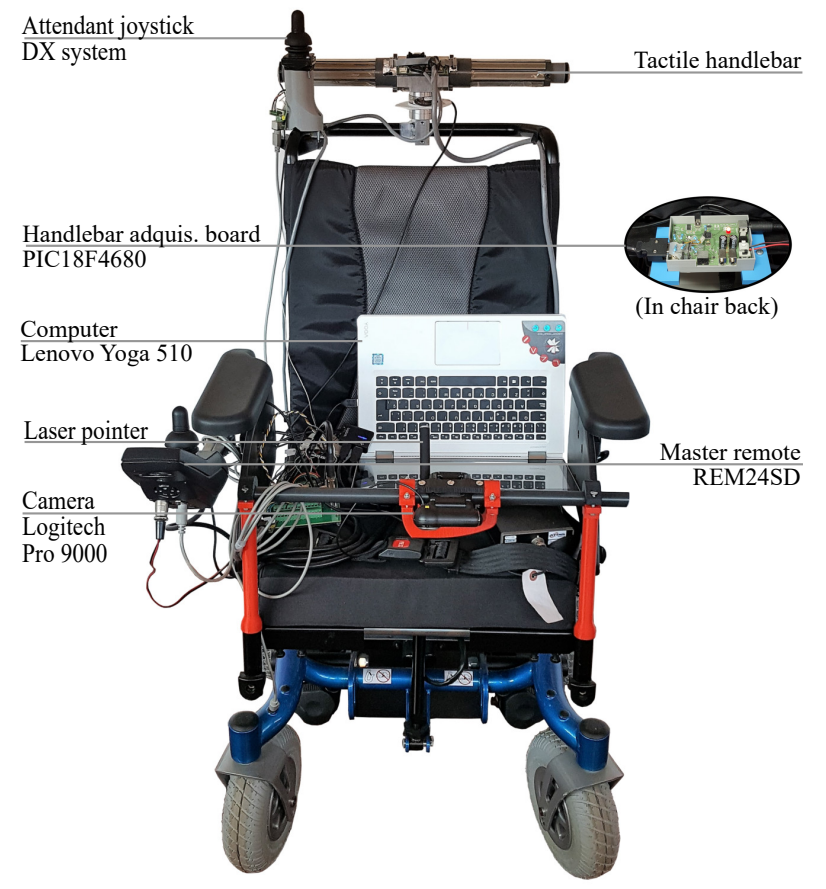

Fig. 6. Experimental setup implementation.

\section{Methodology}

As said above, an experiment was realized to assess the performance of the haptic handlebar and to compare it with the commercial attendant joystick. Besides, a questionnaire aimed to gather the perception of the assistants after driving with both systems was designed.

\section{A. Participants}

Ten volunteers (P1-P10) took part in the experiment, given writing consent and complying with the declaration of Helsinki at all times. They were not aware of the experiment purpose. Among the participants there were $\mathrm{PhD}$ students, professors, laboratory technicians, administrative staff and people outside the university. Their background was varied covering from computer science or electronics to maths, biology, or no higher education. With respect to their previous experience, eight of them had already used a joystick before and none had used formerly a device similar to the assistive handlebar. One of them had experience helping people whose wheelchair has to be propelled by another person. Some statistical measures of the participants' age (in years): Mean $=45.4, S D=9.7$, Max=64 and Min=29.

\section{B. Experiment}

The experiment was performed in two different locations. One of them was the ground floor of an university building. It was an ample space where the volunteers first drove the PW freely with both steering systems for about two minutes. It was intended to emulate the real life situation of driving in an open space with few obstacles (for example, in a park or a pedestrianized avenue). Besides, during this time, some parameters such as the velocity gains or the handlebar height was adapted to the user that needed it. After that, the participants were asked to follow a $35 \mathrm{~m}$ path marked on the ground with a $5 \mathrm{~cm}$ wide yellow tape. As can be seen in Figure 7, it combined straight lines and soft turnings with challenging curves.

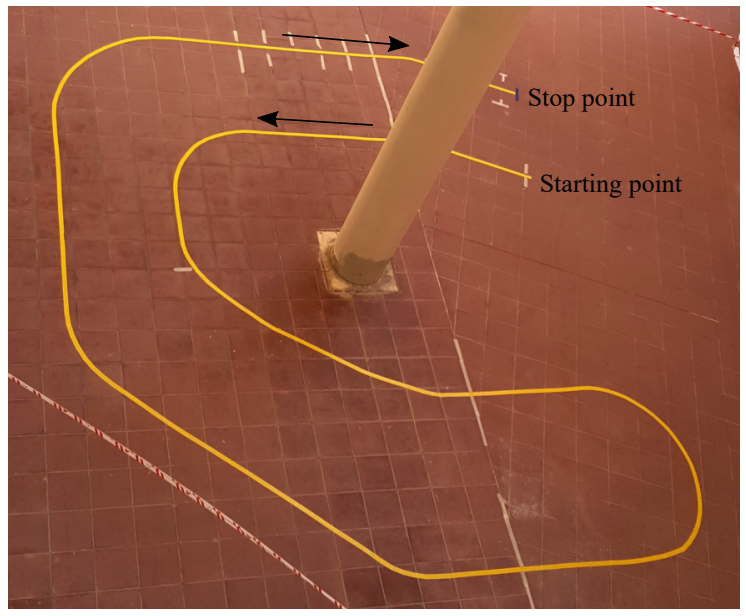

Fig. 7. Path to follow in the experiment.

As said in the previous section, the setup included a laser pointer. It pointed to the ground so that a circular light was visible on the floor just before the chair, aligned with the middle point between the two wheels. During the trial, the participants followed the path, keeping the laser light into the tape to the possible extent but driving as naturally as they 
could. They were not given any instruction about speed or time limitations. They performed the test with one of the devices, and with the other afterwards. Besides, half of the participants started using the attendant joystick and the other half started with the haptic handlebar. The camera added to the setup was also pointing to the ground, aligned with the laser. This way, the experiment realization of every volunteer was recorded for the further estimation of the error between the actual (laser light) and the target (tape) trajectory. The videos were processed in Matlab with color-based segmentation, using Kmeans clustering [38] so that, the tape and the laser light were isolated from the rest of elements of the images, mainly floor tiles and dirt. Figure 8 illustrates this procedure, it shows

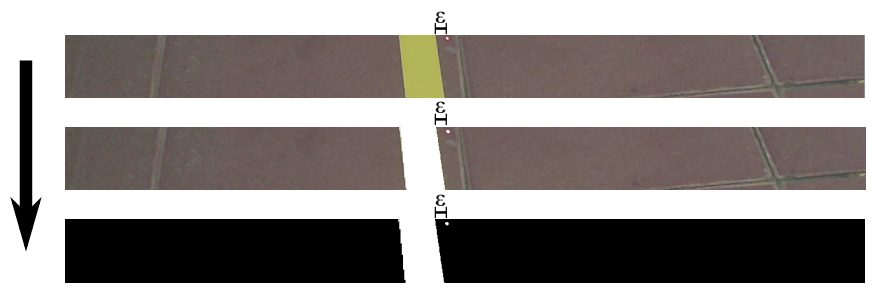

Fig. 8. Color-based segmentation applied to a frame from a sample video.

one sample video frame and the result of being processed to compute the error in following the trajectory. Figure 9 shows the so obtained data of the error registered when the chair is driven with the joystick (left) and with the handlebar (right). Thereafter, the volunteers were moved to an indoor location
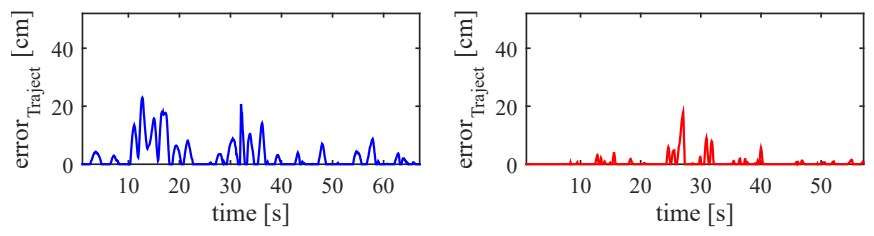

Fig. 9. Error between the actual and target trajectory for one of the participants using the joystick (left) and the handlebar (right).

of the same facility to perform a second trial. It is depicted in Figure 10 and comprises a corridor and a laboratory. If we compare the size of the PW and the available room, we see that the setup implies driving in narrow spaces. The aim was to emulate a daily life driving activity in enclosed and confined places such as the inside of a house, a supermarket, etc. The test consisted in entering the laboratory from the corridor through the door, turning to the left and, once reached certain point, driving backwards arriving at the stop point after turning right. The only marks on the ground were those to indicate the starting and stop points and that where the movement had to be switched from forwards to backwards. Again, the participants did not receive instructions about speed or time, but only about driving as naturally as possible avoiding colliding with the obstacles (walls and table). If they collided they could correct the trajectory as many times as necessary. As with the previous path, half of the volunteers started the test with the joystick and the other half with the handlebar. The number of collisions made with each driving interface was registered.

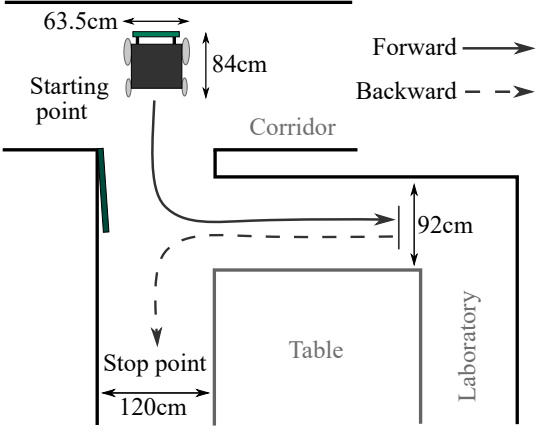

Fig. 10. Corridor and laboratory used in the experiment. Continuous and dashed lines refer to the PW going forward and backward, respectively.

\section{Questionnaire}

After realizing the experiment described just above, the participants were asked to fill a form. They could spend as much time as they needed. The questionnaire was anonymous and the computer screen was placed in such a way that it was not visible for the experiment organizers. A seven-point Likert scale was used to assess both the haptic handlebar and the attendant joystick. Besides, a text entry box was added after every question in case the respondents wished to justify their answer. At the end, they could also leave their general impression of the experience. The content of the form was the following:

Q1. With respect to its use, how do you consider the haptic handlebar / attendant joystick?
1) Very difficult
2) Difficult
3) Rather difficult
4) Neither easy nor difficult
5) Rather easy
6) Easy
7) Very easy

Q2. Concerning comfort, how do you consider the haptic handlebar / attendant joystick?

1) Very uncomfortable

2) Uncomfortable

3) Rather uncomfortable

4) Neither comfortable nor uncomfortable

5) Rather comfortable

6) Comfortable

7) Very comfortable

Q3. How much training do you consider is needed to use the haptic handlebar / attendant joystick?

1) Too much

2) A lot

3) Quite a lot

4) Neither too much nor too little

5) Little

6) Very little

7) None

Q4. Regarding its usefulness, how do you consider the haptic handlebar / attendant joystick?

1) Very useless 
2) Useless

3) Rather useless

4) Neither useful nor useless

5) Rather useful

6) Useful

7) Very useful

Q5. With respect to safety, how do you consider the haptic handlebar / attendant joystick?
1) Very insecure
2) Insecure
3) Rather insecure
4) Neither safe nor insecure
5) Rather safe
6) Safe
7) Very safe

Q6. Concerning physical and mental fatigue, how do you consider the haptic handlebar / attendant joystick?

1) Very tiring

2) Tiring

3) Rather tiring

4) Neither effortless nor tiring

5) Rather effortless

6) Effortless

7) Very effortless

Q7. Leave, if you wish, an additional comment about your experience after having driven with both devices.

As observed, questions are aimed to assess ease of use, comfort, training, usefulness, safety and fatigue.

\section{RESULTS AND DISCUSSION}

In this section the results of the experiment and the answer of the questionnaire are presented and analyzed.

\section{A. Experiment results}

Some parameters have been calculated from the data of the experiment following the path of Figure 7. They are the mean trajectory error $(\overline{\text { Error }})$, the maximum trajectory error $\left(\operatorname{Error}_{M A X}\right)$, the test duration and the product between the mean trajectory error and the duration of each test $(\overline{\text { Error }} \cdot$ Duration). They are depicted in Figure 11 and are aimed to measure the performance of the volunteers when driving with one interface and the other.

The mean error is a good variable to assess how accurate the trajectory tracking was (Figure 11 top). As can be observed, $\overline{\text { Error }}$ shows better numbers for the haptic handlebar than for the joystick in $80 \%$ of the tests. Besides, the difference is considerably larger in four of the ten tests. Below, in the same figure, we see that in the case of Error $_{M A X}$ the values are more similar. The maximum error was observed when the handlebar was used in half of the tests and using the joystick in the other half. However, the meaning of this parameter is relative. A particular volunteer may produce a large deviation at some point and still have a good performance, if we look at the driving during the whole test. The bar chart below that of $\operatorname{Error}_{M A X}$ in Figure 11 gathers the test durations, i.e. how much time the participants needed to complete the full
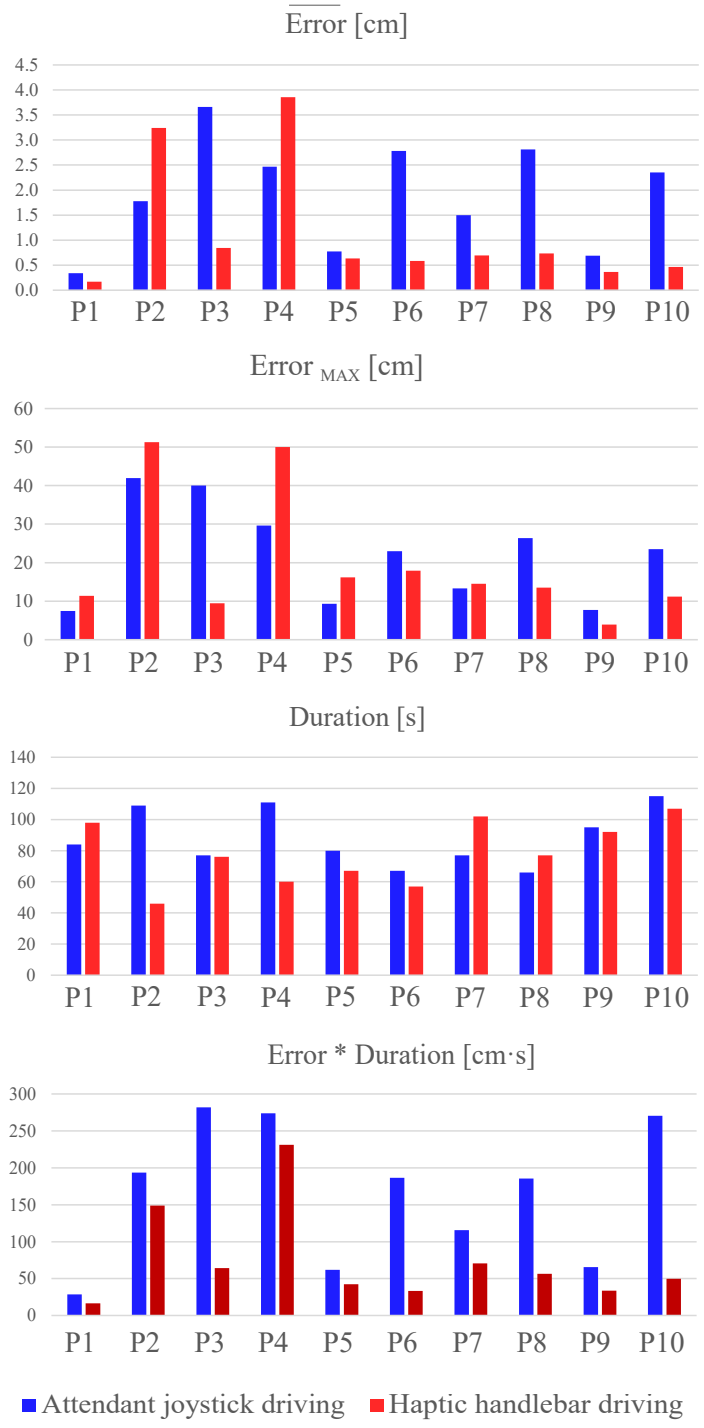

Fig. 11. From top to bottom: mean trajectory error, maximum trajectory error, test duration, product of mean trajectory error by test duration. The results for the attendant joystick are showed in blue and those for the haptic handlebar in red.

path. The required time was longer for the handlebar driving in three of the ten tests. Hence seven participants spent more time following the path when they used the joystick. Finally, in Figure 11 bottom, the bar diagram shows the product $\overline{\text { Error }}$. Duration. This parameter is interesting since the trajectory error and the test duration may be linked. That is to say, if a participant spends much more time undergoing the test with one of the devices than with the other, the error will be probably smaller with the first than with the second. This way, this parameter combines the error and the trial duration so that the latter point is taken into consideration. High values suggest that either long time was taken to carry out the test, or the error made was large or both. On the contrary, behind low values may lie either small errors or little times. In order to distinguish each particular case, $\overline{\text { Error }}$ and duration diagrams are helpful. As observed, the results provided by this parameter are better for the handlebar than for the joystick in all the tests. Focusing on the $\overline{\text { Error }}$ chart (top), P2 and P4 were the 
only two subjects that made a bigger error with the handlebar than with the joystick. However, as said, they got a better $\overline{\text { Error }} \cdot$ Duration. Looking at the Duration bars we find that it could be well due to that fact that they spent about twice the time performing the experiment when the joystick than with the handlebar.

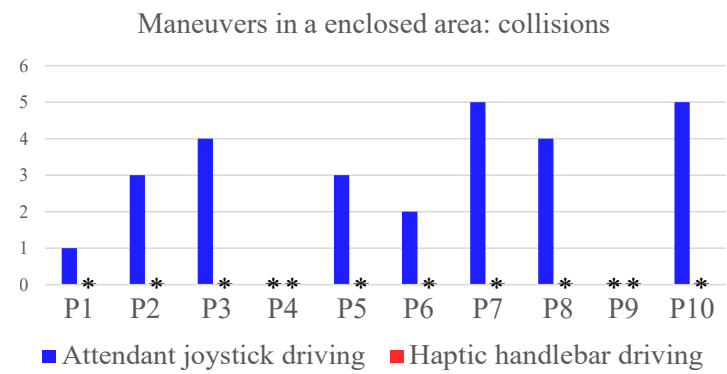

Fig. 12. Number of collisions during driving in the second location with the attendant joystick (in blue) and with the haptic handlebar (in red) (* represents zero collisions)

Regarding the trials performed in the indoor location of Figure 10, the count of collisions with the environment registered for every participant are shown in Figure 12. Collisions took place between the PW and either the walls or the laboratory table. As showed, no impacts occurred when the haptic handlebar was used, in none of the tests. Only in two of the ten tests, there were not impacts when driving with the joystick. In the remaining eight tests, there were between one to five collisions. They occurred mainly in turns while going forward and backward. The backward maneuver was significantly difficult for most of the volunteers. Note that, although time information is not displayed, impacts normally lead to delays and longer times. When the PW collided with an obstacle, it had to be moved away from the latter to resume the march. This process took time. So, driving with the haptic handlebar was faster on more occasions. In addition, the participant with previous experience in caregiving a person who uses a wheelchair is P10. As can be observed in Figure 11 and 12 , his performance is considerable better with the haptic handlebar.

As seen throughout this point, the performance of the haptic handlebar is better than that of the commercial attendant joystick, according to the data extracted during the experiment. However, some unwanted behaviors were also detected. Although there were no collisions, some users realized some of the fine maneuvers with the handlebar producing little tugs, in small discrete movements that are not common in a natural driving. Tugs also appeared for some of them when driving in the location of Figure 7, when the speed was still low at the beginning of the trial. In this case, the phenomenon seemed to be modulated somehow by the gait. It started disappearing as the speed increased. It could be related to a gain too high that makes the system be excessively responsive in situations in which a low velocity is required. Finally, a little asymmetry was observed for two participants. In their case, the system were more sensitive when the handlebar was pushed than when it was pulled. This way, the force needed to make the PW move forward was a little different from that to make it move backward.

\section{B. Questionnaire answers}

Below, in Figure 13, a summary of the responses to the form questions is given. The seven responses available for each question are numbered from the most negative (1) to the most positive impression (7). This way, the higher the number of the chosen option, the better the respondent's opinion is and, conversely, the lower the number associated to the answer, the worse is the participant's perception. The central answer (4) indicates neutrality. Some parameters have been calculated for the groups of responses to each question. They are listed in Table I. The median ( $\tilde{x})$ has been used as measure of the central tendency [39]. In addition, the Mann-Whitney test $U$-value has been computed to assess the statistical significance when comparing the groups of responses given to the same question referring to the attendant joystick and to the haptic handlebar. Finally, another parameter has been computed to evaluate the perception of both devices $(\mathrm{Pe})$. It takes into account the number of times that each option (1-7) has been selected as answer to a particular question, so that it gives an overall picture of the participants' perception for each assessed aspect. It is calculated as:

$$
P e_{i_{A J / H H}}=\frac{\sum_{j=1}^{7} n_{A_{j}} \cdot j}{\sum_{j=1}^{7} n_{A_{j}}}
$$

where $i$ refers to asked question (Q1-Q6). $n_{A_{j}}$ represents the number of times that certain option, $A_{j}$, has been selected by the respondents when answering the question $Q_{i} . j$ varies in the interval [1-7], since seven options are always given. $n_{A_{j}}$ is within the range [0-10], as there are ten participants. Note that $P e$ varies in the range [1-7]. $A J$ and $H H$ means attendant joystick and haptic handlebar, respecively.

$P e_{i_{A J}}$ and $P e_{i_{H H}}$ are useful to compare both driving interfaces. In line with the above, if their value is below 4, the global perception is negative. If it is around 4, the opinion about the device tends to be neutral. Finally, above 4 the perception of the driving system starts being positive. In the top part of Figure 13, we can see what the participants think about the ease of use of both devices. Some of them find the joystick kind of difficult to use, with $P e_{1_{A J}}=3.6$ and $\tilde{x}_{1_{A J}}=3.5$. Only two participants think that this device is rather easy or easy to use. In general, they consider the handlebar easy to use, $P e_{1_{H H}}=5.5$ and $\tilde{x}_{1_{H H}}=6$. None of the subjects answered with a negative option (1-3) with respect to the use of this device. Regarding comfort, we see that the opinions about the haptic handlebar are considerably positive $\left(P e_{2_{H H}}=5.8\right.$ and $\tilde{x}_{2_{H H}}=6$, with all the answers between 5 and 7). The attendant joystick acceptation in terms of comfort has been quite neutral looking at $P e_{2_{A J}}=4.3$, or moderately positive considering $\tilde{x}_{2_{A J}}=5$. Four participants have a perception moderately negative and six moderately positive. With respect to the amount of training required to use properly the driving interfaces, half of the volunteers are neutral (4) whereas the other half have the impression that quite a lot training is needed to use the joystick, $P e_{3_{A J}}=3.5$ and $\tilde{x}_{3_{A J}}=3.5$. The handlebar is perceived as an interface 
for which not much training is required, $P e_{3_{H}}=5.4$ and $\tilde{x}_{3_{H H}}=5$. Concerning the question geared to finding out what users think of the usefulness of both devices, the result is quite significant since both parameters represents a quite positive trend: $P e_{4_{A J}}=5.2, \tilde{x}_{4_{A J}}=5.5, P e_{4_{H H}}=6.5$ and $\tilde{x}_{4_{H H}}=6.5$. It may indicate they perceive the assistance of the PW driving as useful and, in this direction, any help is appreciated. Regarding the sense of safety, the thoughts about the attendant joystick are mainly neutral, $P e_{5_{A J}}=4.1$ and $\tilde{x}_{5_{A J}}=4$, and negative and positive opinions are compensated. If the joystick is not moved the PW is stopped, but if it is pressed there is no way of knowing whether it has been activated involuntarily or by a blow with an object, what may be perceived as risky. Sense of security is greater with the haptic handlebar, $P e_{5_{H H}}=5.4$ and $\tilde{x}_{5_{H H}}=6$, which automatically stops the PW if the user releases the handlebar. Finally, at the bottom, the last question is aimed at the fatigue produced by driving. Regarding the use of the joystick, the overall sensation is neutral, $P e_{6_{A J}}=4.1$ and $\tilde{x}_{6_{A J}}=4.5$, with positive and negative opinions in equal numbers. The handlebar is seen more or less as an effortless device, $P e_{6_{H H}}=5.4$ and $\tilde{x}_{6_{H H}}=6$. Furthermore, all the results of comparing the answers given for the joystick and the handlebar with the Mann-Whitney U Test are statistically significant (the critical value of $U$ at $p<0.05$ is 27).

TABLE I

$P e$ COEFFicient, Median ( $\tilde{x}$ ) AND $U-$ value of MANN-Whitney Test (WITH $U_{\text {critical }_{p<0.05}}=27$, ONE-TAILED) FOR THE LIKERT SCALE RESULTS OF THE QUESTIONNAIRE.

\begin{tabular}{|l|l|c|c|c|}
\cline { 3 - 5 } \multicolumn{2}{l|}{} & $P e$ & $\tilde{x}$ & $U-$ value \\
\hline \multirow{2}{*}{ Q1. Ease of use } & Attendant Joystick & 3.6 & 3.5 & \multirow{2}{*}{12.5} \\
\hline \multirow{2}{*}{ Q2. Comfort } & Haptic Handlebar & 5.5 & 6 & \\
\hline \multirow{2}{*}{ Q3. Required training } & Attendant Joystick & 4.3 & 5 & \multirow{2}{*}{22.5} \\
\hline \multirow{2}{*}{ Q4. Usefulness } & Attendant Joystick & 3.5 & 3.5 & \multirow{2}{*}{2.5} \\
\hline \multirow{2}{*}{ Q5. Safety } & Haptic Handlebar & 5.4 & 5 & \multirow{2}{*}{17.5} \\
\hline \multirow{2}{*}{ Q6. Fatigue } & Attendant Joystick & 5.2 & 5.5 & \multirow{2}{*}{23.5} \\
\hline
\end{tabular}

$P e, \tilde{x}$ perception reference: 1 (Negative) $\leftarrow 4$ (Neutral) $\rightarrow 7$ (Positive)

As said in the previous section, the participants were allowed to leave comments if they wished. Some of them highlight the ease of use of the haptic handlebar: "With a little practice, it's easy to control the movement", "After using it a little, it is easy to handle", "It's easy to adapt to the device", etc. Also its usefulness: "It greatly reduces the effort that has to be made in order to move and steer the chair" or "This device has really ample possibilities". Others may seem to be negative, like the following about safety: "Mainly during the learning period [it is rather insecure]. With practice, it gets safer". Or this one speaking of fatigue: "It can be rather tiring in the beginning when you are focused on the handlebar. After getting used to it a little, it's not tiring at all.". However, when they say "the learning period" or "the beginning" they
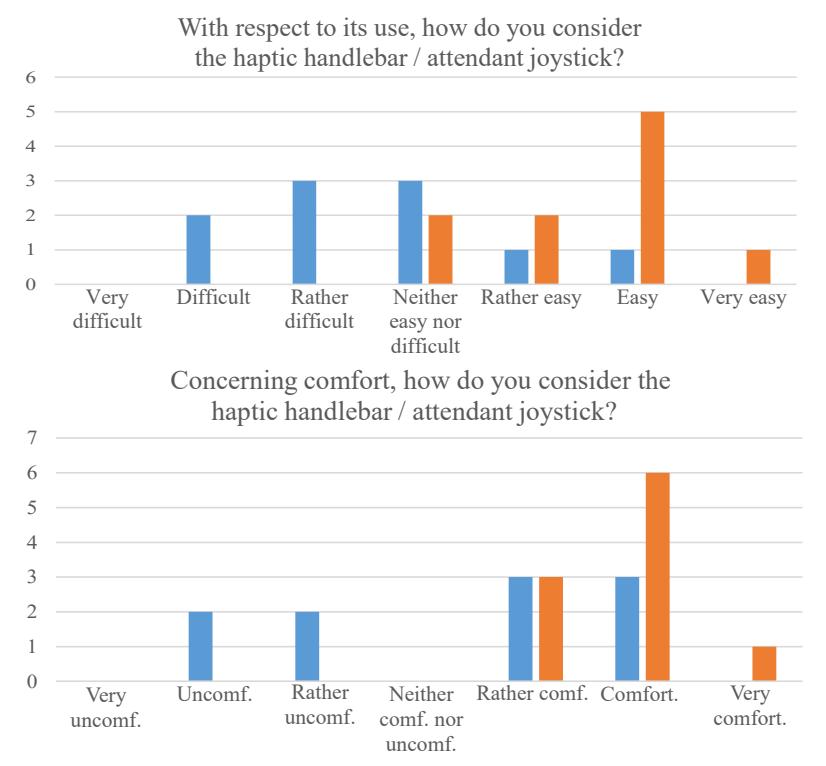

How much training do you consider is needed to use the haptic handlebar / attendant joystick?

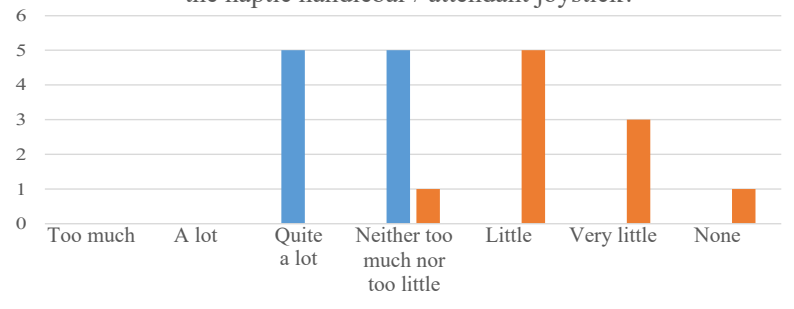

Regarding its usefulness, how do you consider the haptic handlebar / attendant joystick?

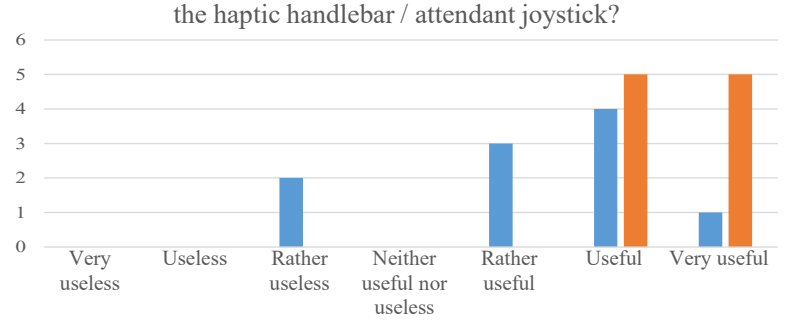

With respect to safety, how do you consider the haptic handlebar / attendant joystick?

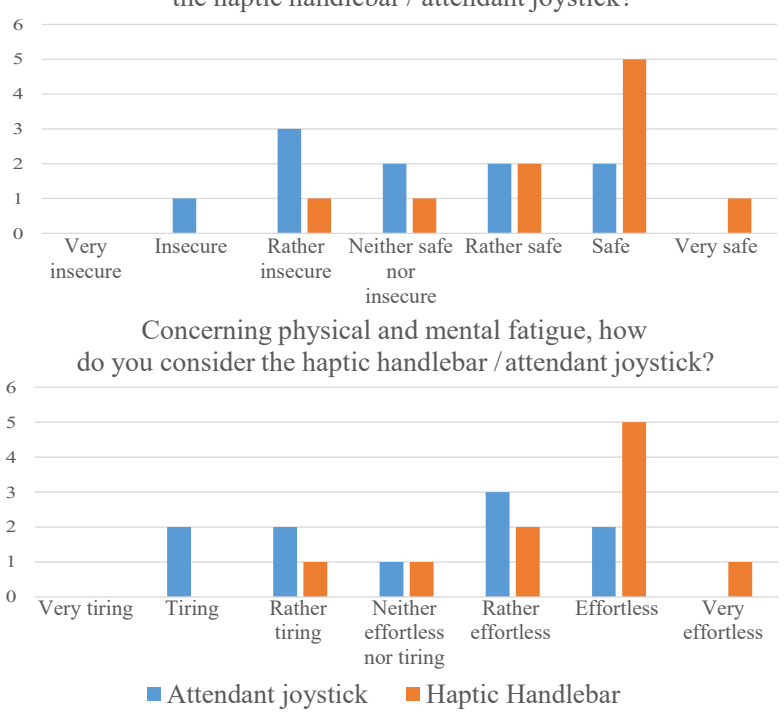

Fig. 13. Participants' opinion about different aspects of the driving with both devices. From top to bottom: ease of use, comfort, training, usefulness, safety and fatigue. 
are referring to their learning period that was no more than two minutes, as explained previously. However, there are also some of them that can help to improve the device. For example: "[...] the system is a little slow at first, when you have to wait to hear the sound signal. What if I needed to move the chair quickly to avoid an obstacle heading towards us?". Note that this volunteer reflects on the hypothetical scenario in which the PW has to be moved while the $C_{o} M_{L_{r}}$ and $C o M_{R_{r}}$ are being computed in the calibration process. It suggests that a secondary "basic" control that allows a range of limited movements (perhaps, moving forward o backward a little) and does not depend on the CoMs could be implemented. It may be used in case of emergency or to move the PW without the purpose of driving it (for example, if it is stopped and is an obstacle in the trajectory of another person).

Regarding the comments about the joystick, those about its use are, for example: "It is operated with only one hand, what I found more complicated and less natural than the handlebar use" or "The lateral movements are abrupt and it is difficult to follow a determine trajectory because of the zigzag. Only the forward movements are steady". They transmit lack of intuitiveness and difficulty in the maneuverability. With respect to comfort, they continue in the same vein: "It is more easy to accommodate and to steer grasping the handlebar that grasping the joystick", "The hand posture is not very natural". In general, they reflect that training is needed: "It requires much more training than the handlebar" or "The backward driving is difficult and it requires considerable training". They also highlight the superiority of the handlebar in safety terms: "(...) I think that the handlebar monitors in a better way what the attendant is doing [grasping monitoring]. Besides, the handlebar has a softer response to certain kind of maneuvers". In summary, the commentaries are on the same line as the rest of results of the experiment.

\section{CONCLUSION AND FUTURE WORK}

This paper has presented a new control strategy for the attendant driving device of a power wheelchair that consists in a haptic handlebar based on tactile sensors. This strategy has been evaluated and compared to the performance of an attendant joystick as the common solution in the marketplace. Two tests have been carried out for this purpose, one obtains data of the error in following a challenging trajectory in an outdoor environment. The second test registers the number of collisions with furniture and doors when another challenging trajectory is followed in an indoor surrounding. Finally, a questionnaire provides subjective information from the participants about the ease of use of the device, comfort, required training, usefulness, safety and feeling of fatigue. The results show a substantial better performance of the haptic handlebar with the proposed control with respect to the joystick, regarding the objective data about error and collisions, as well as user perception. Nevertheless, some aspects can be improved once the results of the tests are analyzed. Firstly, the initial waiting time should be reduced (it lasts about one second currently). It can be reduced with dedicated hardware, and also using user profiles that can be stored in the local memory. Secondly, the control can also be improved in two directions. On one hand, a few users noticed different responses in forward and backward movements, so different gains in the control can be contemplated in these cases for pushing and pulling maneuvers. Moreover, the observed little jerks at very slow velocities can be faced with an admittance control that implements a gain dependent of the velocity [40]. These improvements are planned for future versions of the system.

\section{ACKNOWLEDGMENT}

This work was partially funded by the Spanish Government under contract TEC2015-67642-R, FPU Program and European ERDF funds. It has also been partly accomplished within the laboratory of excellence SMART supported by French state funds managed by the ANR within the Investissements d'Avenir programme under reference ANR-11-IDEX-0004-02.

\section{REFERENCES}

[1] "World Population Prospects: The 2015 Revision," United Nations. Department of Economic and Social Affairs, Population Division, 2015, URL: https://esa.un.org/unpd/wpp/publications/files/key_findings_wpp_ 2015.pdf [accessed: April, 2017].

[2] "World Health Organization. What are the public health implications of global ageing?" 2011, URL: http://www.who.int/features/qa/42/en/ [accessed: April, 2017].

[3] B. A. Hawkins, "Aging well: Toward a way of life for all people," Preventing Chronic Disease, vol. 2, no. 3, p. A03, July 2005.

[4] "Disability statistics - barriers to social integration," EUROSTAT: Statistic explained, 2015, URL: http://ec.europa.eu/eurostat/statisticsexplained/index.php/Disability_statistics_-_barriers_to_social_ integration [accessed: April, 2017].

[5] W. C. Mann, C. Llanes, M. D. Justiss, and M. Tomita, "Frail older adults' self-report of their most important assistive device," OTJR: Occupation, Participation and Health, vol. 24, no. 1, pp. 4-12, 2004. [Online]. Available: https://doi.org/10.1177/153944920402400102

[6] C. A. Cifuentes and A. Frizera, Assistive Devices for Human Mobility and Gait Rehabilitation. Cham: Springer International Publishing, 2016, pp. 1-15. [Online]. Available: http://dx.doi.org/10.1007/978-3319-34063-0_1

[7] W. S. Harwin, J. L. Patton, and V. R. Edgerton, "Challenges and opportunities for robot-mediated neurorehabilitation," Proceedings of the IEEE, vol. 94, no. 9, pp. 1717-1726, Sept 2006.

[8] C. Cans, J. D. la Cruz, and M.-A. Mermet, "Epidemiology of cerebral palsy," Paediatrics and Child Health, vol. 18, no. 9, pp. 393-398, September 2008.

[9] M. Rantakokko, M. Mänty, and T. Rantanen, "Mobility decline in old age," Exercise and Sport Sciences Reviews, vol. 41, no. 1, pp. 19-25, 2013.

[10] L. Robinson, G. Gibson, A. Kingston, L. Newton, G. Pritchard, T. Finch, and K. Brittain, "Assistive technologies in caring for the oldest old: a review of current practice and future directions," Future Medicine, vol. 4, no. 9, pp. 365-375, August 2013.

[11] A. Brandt, S. Iwarsson, and A. Ståhle, "Older people's use of powered wheelchairs for activity and participation," Journal of Rehabilitation Medicine, vol. 36, no. 2, pp. 70-77, March 2004.

[12] L. Fehr, W. Langbein, and S. Skaar, "Adequacy of power wheelchair control interfaces for persons with severe disabilities: a clinical survey," Journal of Rehabilitation Research and Development, vol. 37, no. 3, pp. 353-360, May/June 2000.

[13] C. Torkia, D. Reid, N. Korner-Bitensky, D. Kairy, P. W. Rushton, L. Demers, and P. S. Archambault, "Power wheelchair driving challenges in the community: a users' perspective," Disability and Rehabilitation: Assistive Technology, vol. 10, no. 3, pp. 211-215, 2015. [Online]. Available: http://dx.doi.org/10.3109/17483107.2014.898159

[14] G. Lee, K. Kim, and J. Kim, "Development of hands-free wheelchair device based on head movement and bio-signal for quadriplegic patients," International Journal of Precision Engineering and Manufacturing, vol. 17, no. 3, pp. 363-369, 2016. [Online]. Available: http://dx.doi.org/10.1007/s12541-016-0045-5 
[15] H. Seki and S. Tadakuma, "Straight and circular road driving control for power assisted wheelchair based on fuzzy algorithm," in IECON 2006 - 32nd Annual Conference on IEEE Industrial Electronics, Nov 2006, pp. 3898-3903.

[16] B. E. Dicianno, D. M. Spaeth, R. A. Cooper, S. G. Fitzgerald, M. L. Boninger, and K. W. Brown, "Force control strategies while driving electric powered wheelchairs with isometric and movement-sensing joysticks," IEEE Transactions on Neural Systems and Rehabilitation Engineering, vol. 15, no. 1, pp. 144-150, March 2007.

[17] R. S. Rao, R. Seliktar, and T. Rahman, "Evaluation of an isometric and a position joystick in a target acquisition task for individuals with cerebral palsy," IEEE Transactions on Rehabilitation Engineering, vol. 8, no. 1, pp. 118-125, Mar 2000.

[18] R. K. Megalingam, V. Rangan, S. Krishnan, and A. B. E. Alinkeezhil, "Ir sensor-based gesture control wheelchair for stroke and sci patients," IEEE Sensors Journal, vol. 16, no. 17, pp. 6755-6765, Sept 2016.

[19] A. Fattouh, M. Sahnoun, and G. Bourhis, "Force feedback joystick control of a powered wheelchair: preliminary study," in 2004 IEEE International Conference on Systems, Man and Cybernetics (IEEE Cat. No.04CH37583), vol. 3, Oct 2004, pp. 2640-2645 vol.3.

[20] A. Hadj-Abdelkader, B. Cherki, and G. Bourhis, "Powered wheelchair driving using a $3 \mathrm{~d}$ haptic device," in 2015 International Conference on Virtual Rehabilitation (ICVR), June 2015, pp. 106-114.

[21] V. K. Narayanan, F. Pasteau, M. Marchal, A. Krupa, and M. Babel, "Vision-based adaptive assistance and haptic guidance for safe wheelchair corridor following," Computer Vision and Image Understanding, vol. 149, pp. 171 - 185, 2016. [Online]. Available: http://www.sciencedirect.com/science/article/pii/S1077314216000539

[22] Sugihara, Keisuke, Fujimoto, Shinsaku, and Yoshida, Koji, "Development of electric wheelchair with input of force feedback joystick," MATEC Web of Conferences, vol. 51, p. 02008, 2016. [Online]. Available: https://doi.org/10.1051/matecconf/20165102008

[23] A. Z. Neto, A. Mesquita, M. M. Spindola, and M. Magnani, "Prototype of a wheelchair controlled by cervical movements," in 2014 IEEE International Conference on Bioinformatics and Bioengineering, Nov 2014, pp. 134-140.

[24] N. Peixoto, H. Nik, and H. Charkhkar, "Voice controlled wheelchairs: fine control by humming," Computer methods and programs in biomedicine, vol. 1, no. 112, pp. 156-165, October 2013.

[25] J. Roberts, H. Young, K. Andrew, A. McAlpine, and J. Hogg, "The needs of carers who push wheelchairs," Journal of Integrated Care, vol. 20, no. 1, pp. 23-34, 2012. [Online]. Available: http://dx.doi.org/10.1108/14769011211202265

[26] "Dynamic Controls. DX2 Compact Attendant Remote Module," 2017, URL: https://dynamiccontrols.com/en/mobility-product-users/products/ $\mathrm{dx} 2 /$ secondary-remotes [accessed: January, 2017].

[27] T. Suzuki, H. Uchiyama, C. Holloway, and N. Tyler, "Assisting control for attendant propelled wheelchair based on force velocity relationship," in 2012 Annual International Conference of the IEEE Engineering in Medicine and Biology Society, Aug 2012, pp. 3073-3076.

[28] A. Kakimoto, H. Matsuda, and Y. Sekiguchi, "Development of powerassisted attendant-propelled wheelchair," in Engineering in Medicine and Biology Society, 1997. Proceedings of the 19th Annual International Conference of the IEEE, vol. 4, Oct 1997, pp. 1875-1876 vol.4.

[29] H. Kitagawa, K. Terashima, T. Miyoshi, J. Urbano, and S. Nishisaka, "Power assist system for omni-directional transport wheelchair using fuzzy reasoning," in Proceedings of the 2004 IEEE International Conference on Control Applications, 2004., vol. 1, Sept 2004, pp. 123-130 Vol.1.

[30] C. Zhu, M. Oda, M. Yoshioka, T. Nishikawa, S. Shimazu, and X. Luo, "Admittance control based walking support and power assistance of an omnidirectional wheelchair typed robot," in 2010 IEEE International Conference on Robotics and Biomimetics, Dec 2010, pp. 381-386.

[31] S. Katsura and K. Ohnishi, "A wheelchair type mobile robot taking environmental disturbance into account," in 7th International Workshop on Advanced Motion Control. Proceedings (Cat. No.02TH8623), 2002, pp. $500-505$.

[32] J. C. Garcia, M. Marron, J. Ureña, and D. Gualda, "Intelligent wheelchairs: Filling the gap between labs and people," ASSISTIVE TECHNOLOGY RESEARCH SERIES, no. 33, pp. 202-209, September 2013.

[33] J. Castellanos-Ramos, R. Navas-González, H. Macicior, T. Sikora, E. Ochoteco, and F. Vidal-Verdú, "Tactile sensors based on conductive polymers," Microsystem technologies, vol. 16, no. 5, pp. 765-776, 2010.

[34] A. Trujillo-León, R. Ady, F. Vidal-Verdú, and W. Bachta, "A tactile handle for cane use monitoring," in 2015 37th Annual International
Conference of the IEEE Engineering in Medicine and Biology Society (EMBC), Aug 2015, pp. 3586-3589.

[35] A. Trujillo-León and F. Vidal-Verdú, "Driving interface based on tactile sensors for electric wheelchairs or trolleys," Sensors, vol. 14, no. 2, pp. 2644-2662, 2014. [Online]. Available: http://www.mdpi.com/1424$8220 / 14 / 2 / 2644$

[36] "Interlink Electronics. FSR408®)," 2011, URL: http://www. interlinkelectronics.com/FSR408.php [accessed: December, 2016].

[37] A. Trujillo-León, W. Bachta, and F. Vidal-Verdú, "Evaluation of tactile sensors as an alternative to force sensors in an assistive haptic handlebar," in 13th Annual International Conference of IEEE Biomedical Circuits and Systems (BioCAS2017), Oct 2017, pp. 224-227.

[38] "MathWorks. Documentation: Color-Based Segmentation Using KMeans Clustering," 2017, URL: https://es.mathworks.com/help/images/ examples/color-based-segmentation-using-k-means-clustering.html [accessed: July, 2017].

[39] S. Jamieson et al., "Likert scales: how to (ab) use them," Medical education, vol. 38, no. 12, pp. 1217-1218, 2004.

[40] M. Spenko, H. Yu, and S. Dubowsky, "Robotic personal aids for mobility and monitoring for the elderly," IEEE Transactions on Neural Systems and Rehabilitation Engineering, vol. 14, no. 3, pp. 344-351, Sept 2006.

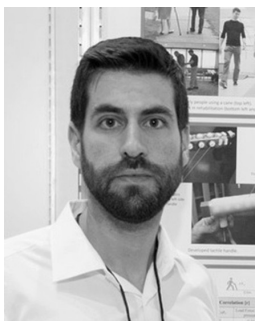

Andrés Trujillo-León was born in La Montiela, Spain. He received the B.Sc. degree in Telecommunication Engineering and the M.Sc. degree in Electronics Engineering from the University of Málaga (UMA), Spain, in 2008 and 2011, respectively. Recently, he received the Ph.D. degree in Mechatronics Engineering from the same university. $\mathrm{He}$ is currently research associate with the group of Electronics for Instrumentations and Systems, Department of Electronics, UMA. His research involves the application of cost-effective solutions in rehabilitation and assistive devices.

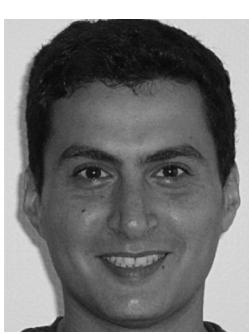

Wael Bachta received the Electrical Engineering degree from the Ecole Nationale Supérieure de Physique de Strasbourg, Strasbourg, France, in 2005, and the M.S. and Ph.D. degrees in robotics from the University Louis Pasteur, Strasbourg, in 2005 and 2008, respectively. He is currently an Associate Professor with the Université Pierre et Marie Curie, Paris, France, where he is a member of the Institut des Systèmes Intelligents et de Robotique. His current research interests include medical robotics and robot control.

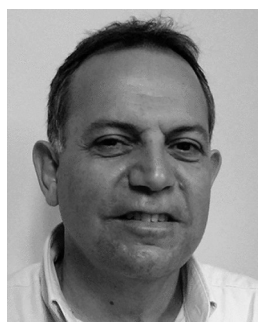

Fernando Vidal-Verdú was born in Cádiz, Spain. $\mathrm{He}$ received the M.Sc. degree in Physics from the Universidad de Sevilla, Seville, Spain, in 1988, and the Ph.D. degree in microelectronics from the Universidad de Málaga (UMA), Málaga, Spain, in 1996. Since 1991, he has been with the Department of Electronics, UMA, where he is currently an Full Professor. He is the author or coauthor of 24 international journal papers, seven chapters of books, and more than 60 conference papers. His current research interests include smart sensors and actuators and specifically in tactile interfaces. 